\title{
EL PENSAMIENTO DIDACTICO DE HANS AEBLI
}

\author{
Ricardo Lucio A. \\ Universidad Nacional de Colombia
}

\section{Introducción}

Algunos pedagogos contemporáneos tratan de deducir de una manera simple y esquemática sus enfoques didácticos de la psicología piagetiena. Su intento se queda cofto, a mi manera de ver, en la medida en que no caen en la cuenta que Piaget no es un pedagogo, sino fundamentalmente un psicólogo evolutivo, un epistemólogo, interesado en construir modelos explicativos del desarrollo de las estructuras mentales en el niño y, con él, de la génesis de la inteligencia. La perspectiva psicológica, como lo reconoce el mismo Piaget, si bien es un punto de referencia útil, es por sí sola insuficiente para generar modelos pedagógicos:

Siempre pensamos que los materiales que nos fue posible reunir con ayuda de numerosos colaboradores, así como las interpretaciones a que este trabajo nos condujo, podrían aportar alguna utilidad pedagógica y, en particular, didáctica. Pero no compete a los psicólogos, cuando no son más que psicólogos, deducir tales consecuencias de sus trabajos, pues aunque conocen al niño carecen de experiencia escolar (Piaget 1962).

Precisamente en ello radica la, importancia de la obra didáctica de Hans Aebli: no sólo porque se formé en la escuela de Ginebra (Piaget fue su director de tesis) y se reconoció siempre como heredero de la línea de pensamiento piagetiana (Aebli fue el editor de la traducción alemana de las obras de Piaget), a pesar de cierto distanciamiento posterior, sino también porque, a diferencia de su maestro, siempre se reconoció como educador, y sus intereses nunca dejaron de ser fundamentalmente pedagógicos "Llegó a nuestro instituto tras brillantes comienzos como maestro de escuela" (Piaget 1962).

Si la teoría piagetiana representa entonces una de las fuentes más importantes de la didáctica de Aebli, su trabajo sistemático en la formación de maestros, en las universidades alemanas y suizas, representa la otra. Su obra se convierte de esta manera en un ejemplo concreto de cómo una didáctica coherente no es ni sólo deducción de la teoría, ni sólo empirismo práctico, sino el resultado de una interacción constructiva entre teoría y práctica: la teoría como marco de referencia y orientación sistematizadora de la praxis, y la praxis como validación, como corrección muchas veces, de la teoría.

Gracias a esta interacción dialéctica la confrontación de Aebli con puntos importantes de la teoría de su maestro se convierte en el impulso inicial para la construcción de su propia didáctica. Al distanciamiento teórico que conllevó esta confrontación está dedicada la primera parte del presente trabajo. La intuición fundamental consistió en descubrir que en las experiencias clínicas piagetianas no solamente tenía lugar la constatación del tipo de estructuras mentales presentes en el niño, sino que también durante ellas se realizaban verdaderos procesos de aprendizaje, en alguna manera orientados por el investigador. $\mathrm{Y}$, si se da aprendizaje, es entonces teóricamente posible, a partir del análisis de dichas experiencias, construir una didáctica.

La segunda parte del trabajo está dedicada a analizar las líneas directrices fundamentales sobre las cuales construye Aebli su didáctica: son ellas la construcción de las acciones, la construcción a partir de ellas de las operaciones, y la construcción operativa de los conceptos. Sin pretender reducir a ellas toda su obra didáctica, sí puede afirmarse que son el andamiaje al que recurre una y otra vez en sus diversos planteamientos 
didácticos. Esta parte se refiere, fundamentalmente, al libro 12 formas básicas de enseñar, recientemente (1988) publicado en lengua española, que sistematiza dos largas décadas de elaboración didáctica y que, a mi manera de entender, es el compendio más completo de la obra didáctica de Aebli.

En la tercera parte, finalmente, recojo algunas observaciones críticas que podrían hacerse a la didáctica de Aebli. De una manera un poco esquemática busco compatibilizar el proceso piagetiano de construcción de las estructuras mentales, con el que proporciona Aebli. Como marco de referencia recurro en el intento a algunos representantes de la psicología soviética (Kostkjuk, Tjlopenkaja, Wygotski y Galperin) que se interesaron por temas similares, y que fueron también manejados por Aebli (él mismo escribió el prólogo a una edición alemana que presentaba algunos trabajos de ellos, Aebli 1969).

La observación que sigue no se acostumbra en este tipo de artículos; a pesar de todo, no quiero dejar de mencionar que este trabajo es un humilde tributo a la memoria de Hans Aebli, muerto en 1990, poco después de haber revisado el manuscrito de la versión al español de su último libro, que tuve el honor de traducir.

\section{Primera parte: el distanciamiento del maestro}

La primera obra importante de Hans Aebli fue su tesis doctoral, dirigida por el propio Jean Piaget en la universidad de Ginebra, y que fue publicada originalmente en francés en 1951: Dídactique psychologique. El libro fue traducido al español en 1958, y es prácticamente el único trabajo en nuestra lengua que se conoció de Aebli, hasta finales de los años ochenta. Por las circunstancias en que fue escrito, pertenece todavía íntegramente a la línea del pensamiento piagetiano; sin embargo, ya desde ese momento se pueden entrever los intereses más marcadamente pedagógico-didácticos de Aebli, en contraposición con los psicológico-genéticos de su maestro. Como lo indica el subtítulo del libro (Application á la didactique de la psychologie de Jean Piaget), Aebli realiza un primer acercamiento a la didáctica práctica, escolar, desde el pensamiento psicológico de Piaget.

Ocho años más tarde (1959/60) escribe en alemán la obra über die geistige Entwicklung des Kindes [desarrollo mental del niño]. En ella se encuentran los primeros pronunciamientos críticos con respecto a la teoría piagetiana. Aunque el trabajo puntualiza algunos aspectos teóricos importantes de la psicología genética y del método experimental (clínico) utilizado por Piaget, hay dos características en la crítica de Aebli que conviene tener en cuenta. En primer lugar, más que el mero interés teórico del psicólogo, Aebli asume desde un principio, como perspectiva, el interés del pedagogo preocupado especialmente por las aplicaciones educativas de la teoría. Tal interés, señalado ya por Piaget en el prólogo a la primera obra de Aebli ${ }^{1}$, guía la investigación teórica de la segunda:

El trabajo presente versa sobre un problema parcial de la sicología pedagógica. Investiga al interior del pensamiento infantil el procesamiento de las estructuras lógicas implicadas en las operaciones y conceptos del pensamiento (Aebli 1963).

En segundo lugar, a diferencia de otros planteamientos críticos que se hicieron por esa época a la psicología piagetiana (los de la psicología soviética, por ejemplo), los de Aebli

\footnotetext{
1 “. . . el valor real del hermoso estudio de $\mathrm{H}$. Aebli radica en que proviene de un autor que es a la vez excelente pedagogo y excelente experimentador en psicología propiamente dicha. Llegó a nuestro Instituto tras brillantes comienzos como maestro de escuela". (Piaget 1962). 
no provienen desde fuera sino desde dentro, ya que éste se formó en la misma escuela de Ginebra, en cuyos experimentos participó anteriormente. Más que negar la teoría de Piaget, busca en sus investigaciones ampliarla. Aunque constata las dificultades de aplicación pedagógica de la teoría piagetiana, debe reconocer que de momento no existe otro punto de partida posible:

Hasta hoy no ha encontrado Piaget en su área ningún interlocutor. Su trabajo imponente se asemeja a una cumbre solitaria a la que no llega eco alguno, puesto que no existe en su ámbito otra montaña de altura comparable. Quien quisiera trabajar la problemática del desarrollo de las estructuras mentales no tiene más alternativa que ocuparse de Piaget (Aebli 1963).

Dos temas revisten significado especial para Aebli en esta época: la manera como se construyen las "estructuras cada vez más comprensivas, transparentes y móviles" y "el papel central del hacer en el pensamiento y evolución del niño" (Aebli 1961).

\section{A. La crítica a Piaget (Aebli 1963)}

1) La primera dificultad que encuentra Aebli en la aplicación pedagógica de la teoría del desarrollo de Piaget radica en las características mismas de la investigación genética desarrollada por éste: "[Esta forma de análisis] no proporciona ningún tipo de información sobre los procesos psicológicos, al interior de los cuales se presentan las estructuras investigadas". En pocas palabras, Piaget efectúa el procedimiento clínico a fin de poder comprobar, por medio de experimentos plenamente controlados, si el niño domina ya una determinada estructura, o no. Este es su logro más significativo. Ese procedimiento investigativo no puede sin embargo, a juicio de Aebli, ni determinar cómo ni por medio de qué procesos se ha logrado dicha estructura, ni tampoco precisar en qué medida se debe atribuir este logro a la maduración interior y en qué medida al aprendizaje.

2) Según Piaget, el desarrollo es el movimiento de un nivel estructural al siguiente, de complejidad más integradora. Como los experimentos sólo pueden constatar qué estructuras han sido ya construidas, la construcción misma que ocurre entre los dos niveles sólo puede deducirse extrapolándola "lógicamente" e interpretándola por medio de modelos lógicos (por ejemplo los grupos y las agrupaciones). Para Aebli, sin embargo, esta interpretación lógica es sólo una parte de la explicación del desarrollo humano:

Un problema distinto es el de si el desarrollo intelectual se puede identificar de hecho con estos procesos de construcción. Frente a Piaget, quien ha hecho de esta proposición un axioma fundamental de su trabajo, creemos nosotros que debemos negarla.

3) A los pedagogos se les presenta una dificultad adicional: puesto que estos procesos de construcción no pueden ser observados, tampoco pueden ser objeto de enseñanza. Serían completamente espontáneos:

Según Piaget, los procesos lógicos de construcción, que conducen de una reacción del niño más primitiva manifestada en un experimento anterior, a una reacción más desarrollada manifestada en un experimento posterior, se comportan como procesos de desarrollo, que fluyen en el tiempo que media entre los dos experimentos. Los progresos del desarrollo que suceden en este lapso de tiempo son para él producto de la actividad mental espontánea del niño.

4) Cuando el experimento piagetiano muestra que una persona ha alcanzado en un momento y situación cualquiera un nivel estructural, Piaget asume este resultado como 
definitivo. Aebli encuentra un poco mecánico este carácter definitivo de las estructuras; habla por tanto de "substancialismo de las estructuras mentales" en Piaget:

En primer lugar hay que afirmar que las estructuras en Piaget se encuentran adheridas a una cierta definitividad. Una operación construida una vez, un sistema construido, lo es de una vez por todas.

5) Cada fase de desarrollo se halla ligada para Piaget a una edad determinada del hombre. Aebli cree por el contrario que "en este nexo [edad del niño frente a nivel estructural] actúa toda una serie de factores adicionales [además del tiempo]".

6) Para la afirmación anterior encuentra Aebli una comprobación en la explicación que da Piaget a los "desplazamientos" (decalages). Un desplazamiento ocurre cuando estructuras lógicas del mismo nivel de desarrollo, iguales o semejantes, aparecen parcialmente en diversos momentos del desarrollo del individuo. En el caso del desplazamiento del concepto de invarianza, Piaget explica la contradicción aparente acudiendo a dos tipos de razonamientos:

a) Las estructuras más comprensivas deberían aparecer, "lógicamente", más tarde. De esta manera se presenta la invarianza del peso con posterioridad a la de sustancia.

b) Se dan condicionamientos de tipo perceptivo y representativo; eso hace que el concepto de peso permanezca ligado durante más tiempo a la actividad muscular que el de sustancia, y por lo tanto sea "agrupado" posteriormente.

Aebli fundamenta en estos condicionamientos perceptivos y representativos su afirmación de que se dan otros factores, además del tiempo de las fases y la edad del niño, que determinan la aparición de las estructuras y su nivel.

\section{B. Ampliación del modelo de Piaget (Aebli 1963)}

\section{La esencia del proceso de elaboración}

Aebli afirma que se deberían efectuar análisis adicionales dentro del método clínico, tal como lo realiza Piaget. Tales análisis adicionales deberían fundamentarse, en síntesis, en las siguientes consideraciones básicas:

a) Las investigaciones genéticas no conducen solamente a comprobar la existencia de las estructuras mentales ya construidas, puesto que en las experiencias mismas tienen lugar también verdaderos procesos de elaboración de operaciones y conceptos. Por medio de tales procesos pueden, o bien aplicarse operaciones ya dominadas (reestructuración), o bien construirse nuevas (estructuración). En ambos casos se trata de la derivación (por medio de la construcción y de la diferenciación) de nuevos elementos, de nuevos esquemas.

b) Estos procesos del pensamiento que se generan tienen como punto de partida el planteamiento de un problema, hecho por el investigador:

Consideramos como lo más probable el que los procesos de construcción que conducen a nuevas estructuras apenas se ponen en marcha cuando se plantea el nuevo problema.

Lo cual quiere decir que en las investigaciones genéticas tienen lugar verdaderos procesos de aprendizaje, los cuales son orientados en cierta manera por el planteamiento 
del problema que hace el investigador y están determinados por las condiciones materiales de la acción que se investiga:

Ahora bien, cada proceso de elaboración es un proceso de aprendizaje. Y en cierta medida tenemos ante nosotros todos los rasgos de un proceso clásico de aprendizaje: el planteamiento de un problema o una 'dificultad de aprendizaje', una situación o un objeto en el cual aparece el problema, hasta un investigador, quien orienta y vigila los procedimientos del pensamiento por los carriles que conducen a la operación buscada y con ello a la solución del problema.

c) Puesto que se llevan a cabo procesos de elaboración y aprendizaje en las experiencias clínicas, es entonces posible ampliar el análisis y estudiar dichos procesos y sus elementos. Para una investigación más profunda de los procesos remite Aebli al lector a su primer trabajo didáctico propiamente tal, cuya primera edición estuvo terminada poco antes de 1961, y que abordaremos en la segunda parte. En la discusión teórica que nos ocupa en este momento, y que es la planteada en el libro del "desarrollo mental del niño", Aebli busca más bien fundamentar la posibilidad de ese análisis didáctico sugerido por su consideración del método experimental piagetiano, y para ello presenta los elementos (factores) del proceso de elaboración de las estructuras mentales:

Este trabajo se plantea como una tarea investigar algunos factores en el proceso de elaboración, que determinan el límite superior de las estructuras lógicas alcanzadas, límite que el niño con el transcurso del tiempo va colocando cada vez más lejos y es por tanto característico de su desarrollo mental.

\section{Los factores del proceso de elaboración}

Según Aebli, el nivel de desarrollo de una estructura lógica que se puede lograr en una fase determinada de la evolución mental depende de seis factores. Los factores pueden ser analizados en una investigación corriente sobre algún tipo de operación efectuada por un niño. Los tres primeros factores se refieren a las condiciones del objeto de la acción, tal como lo había ya mencionado Piaget en su explicación de los desplazamientos; estos son 1) la cantidad de partes del objeto, $\mathrm{N}-$ ' ; 2) la complejidad de éste, Cob; y 3) su evidencia, Eob - Dos factores adicionales se refieren a la acción misma que se realiza en el experimento; 4) los procesos de aprendizaje realizados, A; y 5) la participación de la percepción y la representación en la solución del problema, P. El último factor 6) es la motivación del sujeto de investigación, Mot

El nivel de desarrollo de la estructura de la operación, Estop, se define entonces como una función de estos factores:

$$
\text { Estop }=\mathrm{f}[\mathrm{N}-1, \mathrm{Cob}, \text { Eob, } A, \mathrm{P}, \mathrm{Mot}]
$$

El nivel de la estructura junto con los seis factores determinan lo que Aebli llama "el grado de dificultad del proceso de elaboración" o "la potencia mental que se manifiesta en la elaboración lograda" (PM):

$$
\mathrm{PM}=\text { Estop. } \mathrm{N}-1 \text { Cob. 1/Eob } \cdot 1 / \mathrm{A} \cdot 1 / \mathrm{A} .1 / \mathrm{P} .1 / \mathrm{Mot}
$$

Esta potencia mental, o "la capacidad de solucionar determinadas tareas" es por tanto para Aebli el indicio del desarrollo mental, ya que ella "representa una magnitud constante 
en un momento dado del desarrollo del niño". Diferentes magnitudes de esta potencia mental corresponden por tanto a diferentes fases del desarrollo.

Con ello se pueden explicar más fácilmente los desplazamientos: la variación en un factor dado (o de una combinación de ellos) puede hacer que la magnitud de PM varíe, sin que tenga que variar necesariamente el nivel mismo de la estructura. En otras palabras, en fases diferentes de desarrollo (representadas por valores distintos de PM) pueden aparecer estructuras de nivel similar (representadas por valores iguales de Estop).

Queda también claro que la fórmula tiene validez como una ampliación, y no como una refutación del modelo de Piaget: si se controla el valor de los seis factores manteniéndolos constantes (que es lo que, entre otras cosas, hace Piaget en su método experimental), aparece el nivel de desarrollo mental (PM) como una función de la estructura lógica de las operaciones (Estop).

El encadenamiento de los factores en una fórmula llevaría a sospechar que Aebli se encuentra más del lado de los métodos cuantitativos de la escuela americana y de las teorías en torno a los tests de inteligencia, que del análisis cualitativo de la metódica piagetiana. Sin embargo, parece más bien buscar una síntesis de las dos aproximaciones.

Sin excluir en el que en un futuro dichos factores puedan ser verdaderamente medidos y establecer mediante su multiplicación el valor de PM, la relación contenida en' la fórmula debe interpretarse más bien en el sentido de una multiplicación lógica, que indica simplemente qué factores se encuentran en relación directa (Estop, $\mathrm{N}-1$, Cot) y cuáles en relación inversa (1/Eob, 1/A, 1/P. 1/Mot) con respecto al valor de la potencia mental.

Con la fórmula se corre, sin embargo, el peligro de contemplar el desarrollo mental como un continuo, en vez de hacerlo por etapas hasta cierto punto discretas, como lo propone Piaget. Con ello la diferencia de una fase a otra en el desarrollo del niño sería puramente cuantitativa. Aebli intenta obviar este problema, al señalar cómo diversas magnitudes de los factores pueden estar también caracterizadas por rezagos cualitativamente diferentes:

Con ello se puede resolver de manera impecable la cuestión sobre la continuidad o discontinuidad del desarrollo mental. Todo está a favor de la suposición de que la potencia mental del niño se aumenta de manera continua. Pero las dimensiones en las que pueden manifestarse, los factores que determinan la dificultad de una tarea, no necesariamente representan magnitudes continuas.

\section{Segunda parte: temas centrales del pensamiento didáctico de Aebli}

El trabajo didáctico más importante de Aebli es su libro Grundformen des Lehrens [formas básicas de enseñar]; aunque su primera aparición data de 1961, es fácil deducir de la fecha que lleva la introducción ("Zürich, Año nuevo de 1959"), que Aebli se ocupó simultáneamente de sus primeros enfrentamientos teóricos con la doctrina de Piaget, mencionados en la parte anterior, y del inicio de la construcción de su propia didáctica, representado en este libro. El texto sufrió un proceso de continua reelaboración en su contenido a través de ediciones sucesivas durante los años sesentas y setentas (la 1 la. edición alemana fue publicada en 1978); este proceso refleja, por tanto, que el pensamiento didáctico de Aebli sufrió un proceso de maduración de al menos dos décadas. 
El libro fue dedicado a la formación de maestros; no se trata sin embargo de una mera colección de consejos prácticos que le permitan al maestro dominar diversas situaciones de clase. El análisis de cada "forma de enseñanza" se divide en dos partes: primero se fundamenta en unos principios psicológicos, constructivistas (parte psicológica), para pasar luego a la aplicación sistemática, práctica, de dichos principios en el quehacer del salón de clase (parte didáctica).

Con la experiencia recogida en las ediciones sucesivas de "Formas básicas de enseñar" (que son indicio tanto de la aceptación de la obra como del esfuerzo continuado por mejorarla y reelaborarla), Aebli inicia en la década de los ochentas un trabajo más sólido de sistematización y ampliación del contenido, en una obra didáctica prevista en tres tomos.

El primer tomo apareció en 1983 con el título de Zwölf Grundformen des Lehrens ${ }^{2}$. En este caso no se trata de una edición más del libro anterior: a diferencia de las ediciones anteriores, en las bibliografías corrientes se le cita como un título nuevo, un libro diferente. Además de que se incluyen nuevas "formas", se renueva su estructura global: las "formas" son reagrupadas en dimensiones que corresponden a una nueva fundamentación teórica. Aunque esta estructura nueva es todavía didáctica (es decir, una estructura de la enseñanza), las dimensiones son simultáneamente dimensiones de la construcción del pensamiento, y por lo tanto también formas del aprendizaje. En las ediciones anteriores, aunque cada "forma básica" se encontraba individualmente fundamentada, no estaba sin embargo tan clara su relación (su dimensionalidad) con el conjunto.

El segundo tomo, aparecido en 1987, con el título de Grundlagen des Lehrens ${ }^{3}$, recoge algunas digresiones que habían ido apareciendo intercaladas en las ediciones anteriores de "Formas básicas", para presentar un material algo heterogéneo, que complementa la didáctica básica en temas tales como el aprendizaje social, el aprendizaje autónomo, la teoría curricular, la motivación y la evaluación del aprendizaje. Un tercer tomo debería versar sobre algunos tópicos de las didácticas específicas, tales como la enseñanza de la lengua materna, de las matemáticas, de las ciencias sociales. Sin embargo, aunque Aebli ya había recopilado algunos materiales, su muerte ocurrió en 1990 , sin que pudiera completar el tomo.

Esta estructuración del pensamiento didáctico es comprensible, si se tiene en cuenta que estuvo antecedida por la publicación de otra en dos tomos, Denken: das Ordnen des Tuns [el pensamiento como orden en el hacer] (Aebli 1980 y 1981), en el cual Aebli trabaja de manera más profunda y completa su sicología cognitiva. En él busca el autor sistematizar no sólo sus reflexiones teóricas sobre la problemática del pensamiento, iniciadas con la publicación del "desarrollo mental del niño" (Aebli 1963) y con sus primeros distanciamientos de la teoría piagetiana, sino también sus incursiones en los nuevos desarrollos de las disciplinas emparentadas, especialmente en los campos de la sicología cognitiva norteamericana, la lógica proposicional y la teoría de los computadores. Según su opinión, esta obra de dos volúmenes cristaliza también una década de trabajo científico (Aebli 1980).

En el análisis de los elementos conceptuales claves de la didáctica de Aebli me referiré, fundamentalmente, al primer tomo (el más elaborado) de su didáctica práctica (12

\footnotetext{
${ }^{2}$ Sttutgart, Klett, 1983. Traducción al español: 12 formas básicas de enseñar, Madrid, Narcea, 1988.

${ }^{3}$ Stuttgart, Klett-Cotta, 1987. Traducción al español: Factores de la enseñanza que favorecen el aprendizaje autónomo, Madrid, Narcea, 1991.
} 
formas básicas.., Aebli 1983) y a la obra más teórica, Denken: das Ordnen des Tuns (tomos I y II, Aebli 1980 y 1981). Las dos obras son por tanto paralelas en su confección y complementadas en su enfoque: teoría y praxis, sicología teórica y didáctica aplicada. Al mismo autor le ocurre con frecuencia que, si quiere fundamentar más profundamente determinados pasajes de 12 formas..., debe referirse a los capítulos pertinentes de Denken...

Volviendo a las 12 formas..: las dimensiones en las cuales agrupa Aebli las doce formas básicas de enseñar son tres, llamadas por él "dimensiones de la enseñanza y del aprendizaje", y que corresponden así mismo a tres dimensiones de la competencia didáctica. La primera dimensión son los diferentes medios, en los que se puede realizar el proceso de comunicación; contiene las formas básicas "narrar y referir" (1), "(de) mostrar" (2), "contemplar y observar" (3), "leer y comprender textos" (4). La segunda dimensión se refiere al contenido estructural del proceso mismo de enseñanza/aprendizaje, el cual se construye por medio de "acciones" (forma 6), "operaciones" (forma 7) y "conceptos" (forma 8). La tercera dimensión contiene las diferentes funciones del proceso que posibilita esta construcción: "solución de problemas" (9), "elaboración" (10), "ejercicio y repetición" (11) y "aplicación” (12).

Un primer análisis individual de tales dimensiones permite encontrar ciertos nexos con algunos desarrollos de la psicología cognitiva. Existe, por ejemplo, algún parentesco entre la primera dimensión de Aebli, la de los medios, y el papel del lenguaje en los psicólogos soviéticos, ya que el proceso de enseñanza/aprendizaje solamente puede ocurrir dentro de un proceso de comunicación. También se podría hacer referencia a la obra de Piaget, como el mismo Aebli una vez lo sugirió, con relación al papel que juegan tales medios en la presentación de objetivos "de aprendizaje" y de información por parte del investigador hacia el sujeto de la investigación (el niño), en las investigaciones clínicas. En cuanto a la segunda dimensión (la estructura: acciones, operaciones, conceptos), donde se dejan ver con más claridad las huellas de la enseñanza piagetiana, no sólo hay nexos con la escuela de Ginebra, sino también con el modelo de Wygotski, sobre la construcción de seudoconceptos y conceptos genuinos, y sobre el pensamiento complejo. La solución de problemas de la tercera dimensión, punto de partida necesario en la construcción de las estructuras del pensamiento, se halla presente en las diferentes corrientes de la didáctica moderna y, como en las anteriores, pueden verse en ella herencias de Bruner. La profundización, el ejercicio y la aplicación corresponden, por su lado, a elementos tradicionales de la didáctica.

Pero las dimensiones son también convergentes, no sólo en la estructura de su presentación, sino también en su funcionamiento: dentro de la concepción de Aebli, una estructura específica de la construcción del pensamiento (segunda dimensión) es procesada simultáneamente en un medio concreto (primera dimensión) y en una función concreta del proceso (tercera dimensión). Por eso un análisis individual debe dar paso a un análisis de su conjunto.

Desde este punto de vista, la segunda dimensión se convierte en una especie de núcleo o de infraestructura de la estructura global de 12 formas..., y por tanto de la didáctica de Aebli. Gracias a este núcleo su didáctica se apoya en una teoría constructivista que, aunque implícita, no aparece del todo desarrollada en sus anteriores trabajos didácticos (tampoco en las ediciones anteriores de "Formas básicas..."). El análisis que sigue versa entonces fundamentalmente sobre los tres componentes (las tres formas básicas) de la segunda dimensión; se presenta fundamentalmente una lectura crítica al libro didáctico 12 formas... pero, en la medida en que se requiera una 
fundamentación más profunda y comprensiva en el contexto teórico global, se hace referencia, en puntos específicos, a la obra teórica de base, Denken...

\section{A. La construcción de las acciones (Aebli 1983)}

Las formas más primitivas y sencillas del comportamiento racional son para Aebli las acciones. En este punto de partida coincide con los desarrollos recientes de la psicología cognitiva, y con los de la mayor parte de la evolutiva: "las acciones van más allá de las destrezas: se trata de procedimientos teleológicos, comprensibles en su construcción interna, que producen un resultado captable".

¿Cómo se originan dichas acciones? Según el libro 12 formas..., un intento de respuesta caería aparentemente en un círculo vicioso: una acción es la realización, la aplicación a una situación nueva, de un esquema de acción previo. Pero los esquemas de acción se derivan a su vez de la interiorización de acciones materiales anteriores. Para salir de este círculo (la acción vs. el esquema) hay que reconocer con la psicología genética que en los orígenes de la evolución del pensamiento se encuentran acciones totalmente simples y materiales, apenas diferenciables de los ritmos biológicos.

En el primer capítulo de Denken..., Aebli clarifica ciertos conceptos básicos que empleará a lo largo de la obra, y que nos permiten aquí situar de una manera un poco más precisa el concepto de acción:

1) El comportamiento comprende todos los procesos biológicos (se podría por tanto añadir que este "comportamiento" de Aebli se halla regulado por las leyes piagetianas del desarrollo, la asimilación y la acomodación referidas al equilibrio).

2) El obrar es la actividad humana, el comportamiento consciente.

3) Las dos clases de actividades más importantes son la percepción y la acción.

La acción es por tanto un tipo de obrar (actividad) y de comportamiento. En consecuencia también está regulada en su origen y desarrollo por las leyes del equilibrio, ya que no es otra cosa que una respuesta del individuo frente a su medio, y que tiene como características más sobresalientes la conciencia y la finalidad (e.d. como respuesta a un problema, a una situación de desequilibrio).

Cinco rasgos adicionales completan este cuadro de las acciones:

1) Las acciones son "las formas originarias de la vida intelectual" (Aebli 1983), "tanto en la historia individual del niño, como en la historia de la humanidad". Lo cual significa que todos los procesos cognitivos, el pensamiento y la capacidad reflectiva se derivan de las acciones. Los procesos cognitivos "aparecen dentro del marco de las actividades perceptuales y de la acción", "tienen por tarea reforzar y continuar la construcción de su estructura, y elaborar nuevas estructuras de acción y percepción" (Aebli 1980). En otras palabras: el hombre piensa, porque primero actúa; el hombre estructura sus acciones (por medio de esquemas de acción), porque primero ha comenzado a actuar. "El pensamiento, la reflexión, es por tanto, una metaactividad de las acciones concretas” (Aebli 1980).

2) Las acciones poseen una estructura interna, llamada por Aebli "esquema de acción", a la cual atribuye tres propiedades fundamentales: estos esquemas son a) almacenables como un todo, b) reproducibles y c) aplicables a situaciones nuevas. 
Esto significa, en primer lugar, que el almacenamiento acumulativo de esquemas de acción conforma progresivamente el "saber actuar" (el conocimiento práctico) del individuo $y$, en segundo lugar, que el actuar es flexible, ya que unos mismos esquemas de acción pueden ser realizados en diferentes clases de acciones (efectivas o representadas).

En esta concepción podría existir el peligro del substancialismo, si se llegara a pensar en los esquemas de acción como en una especie de entes con existencia propia, fuera de las acciones mismas. Se trata, irónicamente, del mismo substancialismo que criticaba Aebli en el estructuralismo piagetiano, pero que no se halla muy distante del apriorismo de la filosofía kantiana, a la cual coloca Aebli entre los fundamentos de su credo filosófico (Aebli 1981). El substancialismo se obvia en la medida que los esquemas son considerados, dentro de la enseñanza de Aebli, simplemente como configuraciones estructurales que prestan coherencia al actuar humano. Se evita así el substancialismo, pero no se cae en el polo opuesto del reduccionismo: un esquema puede ser realizado en acciones diferentes (por tanto no se identifica con éstas), pero no puede ni existir, ni desarrollarse independientemente de las acciones y de su sujeto.

3) Aunque Aebli no lo menciona explícitamente, es obvio que, si las acciones poseen una estructura, ésta también puede ser formalizada. Sin ello sería imposible el estudio del actuar humano. Piaget formalizó las operaciones por medio de los grupos y las agrupaciones. Aebli, por su parte, usa la "gramática de casos" de Fillmore (Aebli 1980) y en general el lenguaje de la lógica proposicional, a fin de representar los pasos lógicos consecutivos de un esquema de acción.

4) El corolario más importante de esta teoría de las acciones consiste en que, aunque el hombre por naturaleza (o sea espontáneamente) actúa, sin embargo el actuar, su planificación y su realización, pueden y deben ser aprendidos. Y este aprendizaje es progresivo. Lo cual significa, también, que se dan diferentes niveles en el actuar, según sea la complejidad y el grado de interiorización de las acciones correspondientes.

5) Si existe un aprendizaje de las acciones, puede consecuentemente existir una didáctica de las mismas, y éste es un punto crucial en la doctrina de Aebli (frente, por ejemplo, a Piaget). Desde el punto de vista didáctico el punto de partida de las acciones será siempre el planteamiento y la solución de problemas proceso en el cual se fija un objetivo a la acción.

\section{B. La construcción de las operaciones}

El tema de las operaciones es, quizá, el más importante en la fundamentación conceptual y práctica de la didáctica de Aebli. En su tratamiento se c'dñcentran la mayor parte de los enfrentamientos con las posiciones piagetianas. No obstante, el manejo teórico que hace de ellas Aebli precisa de algunas puntualizaciones que, en mi opinión, no están del todo claras en sus textos; gracias a ello es posible, mediante una confrontación de las posiciones de Piaget y Aebli, esbozar un modelo que las integre a ambas.

Comienzo entonces por examinar lo que dice el propio Aebli con respecto a las operaciones, paso en seguida a manifestar mis propias dudas teóricas con respecto a esas concepciones, para clarificarlas luego por medio de textos adicionales suyos. Podré finalmente establecer qué acerca y qué separa a los modelos de Aebli y de Piaget. 


\section{1) Los planteamientos de 12 formas... (Aebli 1983)}

Las operaciones son acciones abstractas (o "abstraídas") y no necesariamente acciones interiorizadas. Se pueden realizar diferentes tipos de acciones: materiales o representadas, efectivas o interiorizadas. Un tipo cualquiera de estas acciones (o sea, no necesariamente de las interiorizadas) se convierte en operación, cuando tiene lugar el proceso de la abstracción. ¿Qué se abstrae? La coherencia interna de los componentes de la acción, su estructura intrínseca, sus relaciones inherentes. Del acápite anterior se concluye que, según Aebli, cada acción posee una estructura (el esquema de acción). Y es precisamente el tener en cuenta dicha estructura, por parte del sujeto de la acción, más que el tipo mismo de la acción (interiorizada o no), de sus componentes o de su representación, lo que constituye la característica esencial de las operaciones. Este concepto simple de operación es repetido varias veces en Denken...:

Una operación se distingue de una acción ante todo por la manera de dirigirla o, expresado más sencillamente, por aquello que tiene en cuenta el sujeto durante su ejecución (Aebli 1980).

La mención algunas características adicionales de la operación sirve para profundizar su comprensión teórica:

a) Las operaciones son móviles. Esto significa, por una parte, que son construidas y por tanto que sólo pueden ser entendidas por medio de un modelo constructivista y, por otra, que las operaciones son complementadas por otras de nivel superior, al conformar sistemas de complejidad creciente.

b) Las operaciones pueden ser codificadas mediante símbolos de abstracción creciente, o pueden ser sencillamente pensadas de manera intuitiva. La simbolización de las operaciones, que está directamente relacionada con el proceso de abstracción, posibilita su interiorización.

c) Esta traducción de operaciones a signos puede también automatizarse. Como lo reconoce cualquier psicólogo del comportamiento, el proceso de automatización significa ahorro en tiempo y energía; sin embargo existe el peligro de que se confunda la operación misma con la automatización de sus signos o de sus fórmulas, lo cual tiene consecuencias didácticas serias.

Tal como en el tratamiento de las acciones, la aplicación didáctica de la teoría de las operaciones parte también del principio de que el operar es aprendido y enseñado. Para mejor comprender a Aebli en este punto conviene distinguir entre el "operar" y la "operación", entre el proceso y el momento. En primer lugar "operar", como proceso racional, es lo que puede ser aprendido; como proceso dinámico tiene un punto de partida (el planteamiento de problemas), un desarrollo, una profundización y unas posibilidades de aplicación. Todos éstos son subprocesos que son analizados en las correspondientes "formas básicas" de la didáctica de Aebli; en ellos lo más importante es que el "operar" sólo puede ser construido teniendo en cuenta la acción correspondiente (realizada simultáneamente o representada); cuando no se llena este requisito no se "opera", por más que se aprenda de memoria y se repita, por ejemplo, una fórmula automatizada. En segundo lugar, la "operación" misma, como momento estructural, es difícil de conceptualizar; la conciencia de las relaciones internas, que caracteriza una operación, puede lograrse en un momento del todo impredecible: es la intuición, la captación repentina, la construcción de la estructura de coherencia de una acción, en la cual según Aebli se puede prestar ayuda externa, la cual también se puede representar simbólicamente, 
pero la cual sigue siendo, con todo, el logro totalmente propio del actuante (que se convierte de este modo en operante). Una tercera cosa es el "resuh tado" externo de la operación: puede lograrse por medio de un proceso automatizado; la intuición por el contrario es el momento de la claridad, en el cual el sujeto (re) construye la operación; es un acto de la inteligencia.

\section{2) Las contradicciones en la definición de operación}

De las características de la acción anotadas en el numeral anterior se podría quizá concluir que una acción cualquiera puede convertirse en operación (o mejor: que a partir de una acción cualquiera puede originarse una operación), cuando el sujeto actuante tiene presentes las relaciones internas de los componentes de la acción. Con esto tendríamos una concepción comprensiva y amplia del operar, que correspondería a una concepción también comprensiva del actuar. $Y$, en la medida en que veamos más tarde cómo la formación de los conceptos es la prolongación de esta dinámica, podríamos en consecuencia caracterizar fácilmente la tríada acciones-operaciones-conceptos como los momentos esenciales en el proceso de construcción del pensamiento humano, y como núcleo de la enseñanza didáctica de Aebli.

Sin embargo, leemos en la introducción a la forma básica 7, "construir una operación", que "se trata del pensamiento matemático" (Aebli 1983); más adelante se habla así mismo de "conceptos y operaciones matemáticos". Hay que responder entonces al siguiente interrogante: ¿qué entiende Aebli por "pensamiento matemático"? Existen dos respuestas posibles: o bien el pensamiento matemático se refiere al mero medir y calcular (a), o bien significa en general la formalización progresiva del pensamiento, que caracteriza la direccionalidad de su desarrollo y se encuentra por tanto, de maneras diferentes, en cada una de sus etapas (b). Si la respuesta es (a), habría que admitir que solamente determinadas relaciones internas de la acción pueden convertirse en operaciones, con lo cual se reduciría el concepto de operación. Si la respuesta es (b), hay que preguntarse entonces por qué las aplicaciones didácticas y los ejemplos prácticos del operar en la obra de Aebli tienen un criterio tan restringido.

Aebli manifiesta en 12 formas... que el pensamiento matemático es "algo más que solamente calcular": "es un tipo de pensamiento que contempla de manera abstracta la realidad y el obrar propio". A esta afirmación, que podría colocarse dentro del marco de la respuesta (b), se contraponen sin embargo todos los ejemplos concretos de operaciones en la parte psicológica del capítulo, y todas las indicaciones prácticas de la parte didáctica, de las cuales se infiere que el autor, consciente o inconscientemente, se coloca al lado de la concepción limitada de operación (respuesta a):

- Después de haber definido a las operaciones como "acciones abstractas", aporta tres ejemplos. En el primero se trata de una clase cuyos alumnos elaboran un periódico. Es una acción que había aparecido antes como ejemplo para una clase de lenguaje en el capítulo sobre "elaboración de un curso de acción". Allí se discutieron los diversos pasos de esta acción compleja emprendida en la clase: reportes, redacción, publicidad, mecanografía, costeo de la impresión, impresión y administración, etc. Al tratarse ahora de operar, cuando los alumnos deben tener en cuenta las relaciones intrínsecas y la estructura inherente de esta acción, "reconstruyen los alumnos la acción realizada desde el punto de vista de las cantidades y los precios". En los otros dos ejemplos de operaciones es aún más clara esta limitada concepción de la operación: en el segundo "los alumnos de primer grado descubren la medición" y en el tercero "se recogen del depósito 5 x 4 botellas de gaseosa". 
- Los ejemplos de construcción operacional son todavía más dicientes: el aprendizaje de las operaciones aritméticas (adición y multiplicación), el cálculo de la superficie de los rectángulos (este ejemplo ya había sido utilizado en Aebli 1951) y el número PI.

- Cuando habla de la codificación y automatización de las operaciones los ejemplos también tienen siempre alguna relación con números y cálculo numérico.

- La parte didáctica no es una excepción: allí se tiene la impresión de que las indicaciones prácticas se refieren exclusivamente a las clases de matemáticas y geometría.

En todos estos casos prácticos "abstraer" significa aislar los aspectos calculables, medibles, de la acción. Del "algo más que solamente calcular" mencionado en la conceptualización teórica no queda más que, a lo sumo, la posibilidad de captar las relaciones internas de dichos aspectos calculables o aritmetizables de la acción.

Frente a esta concepción estrecha del operar se perfilan con mucha mayor claridad las precisiones del libro Denken...: allí, después de haber definido en el capítulo VII (Aebli 1980) a las operaciones como acciones abstractas y no necesariamente interiorizadas, se ocupa el autor de la clarificación de este proceso de abstracción: significa que las acciones producen relaciones y que se da una "transición por etapas de la acción a la operación".

Hasta ahí no hay ninguna diferencia significativa con la concepción teórica general de operación encontrada en 12 formas...: en general las operaciones son un paso más en la construcción del pensamiento, cualitativamente diferentes de las acciones, pero que se desprenden, se desmiembran, al mismo tiempo de ellas. Este desprendimiento ocurre gracias al proceso de la abstracción.

Poco más adelante, sin embargo, trae Aebli una precisión fundamental: todo lo que hasta ahora se ha dicho pertenece al concepto general de operación, que llama "psicológico". Las operaciones matemáticas o algebraicas (los dos conceptos son usados de una manera indistinta) son sólo una clase, un caso especial, de las operaciones generales, psicológicas:

Ahora, se da no solamente un concepto psicológico de operación, tal como lo hemos usadohasta ahora, sino también uno matemático...

Las operaciones algebraicas son operaciones en el sentido definido por nosotros (Aebli 1980).

Las directrices para la construcción son por tanto válidas para todas las clases de operaciones, y no sólo para las matemáticas, que constituyen un caso particular. La construcción de estas últimas se clarifica por medio de la formalización de la teoría de conjuntos, que debe por tanto corresponder a un caso específico del proceso de abstracción.

En síntesis, la contradicción encontrada en el tratamiento que hace Aebli de la operación radica en limitar, desde el punto de vista práctico-didáctico (en 12 formas. . .), una concepción teórica más amplia y comprensiva (en Denken...). 


\section{3) Las operaciones en Piaget frente a las operaciones en Aebli}

En lo que se refiere a las operaciones, Aebli busca siempre tomar posición frente a su antiguo director de tesis. Coinciden, sin embargo, en dos puntos importantes:

a) las operaciones se originan por medio de "abstracción a partir de la acción", "abstraction á partir de l'action" (Aebli 1980), y

b) las operaciones son móviles, lo cual significa no sólo movilidad entre sistemas operatorios (cada nivel operacional es integrado en un nuevo sistema de mayor complejidad), sino también movilidad en el interior de un sistema: "es la movilidad en relación con sus elementos, con su disponibilidad pronta y segura en el repertorio de los esquemas de acción y de las representaciones materiales" (Aebli 1980).

Las discrepancias más relevantes se concentran, por otro lado, en los cuatro puntos siguientes (Aebli 1980):

a) Las operaciones son para Piaget productos naturales que surgen automáticamente con el desarrollo del pensamiento. Para Aebli son productos culturales, que deben ser aprendidos

b) "La transición por etapas de la acción a la operación" significa para Aebli que las acciones se van operacionalizando. "Esta noción nos separa radicalmente de Piaget, para quien la operatividad representa un fenómeno de todo-o-nada [...]".

c) Para Aebli la asociatividad y la reversibilidad de las operaciones constituyen solamente un caso específico de la movilidad del pensamiento. Otra cosa ocurre, según Aebli, con Piaget: "La movilidad del pensamiento es, según él [Piaget], expresión de la reversibilidad y de la asociatividad en estructuras con forma de grupo".

d) Como consecuencia, los grupos y las agrupaciones representan para Aebli solamente una forma especial de las operaciones: "algunas de ellas pueden coordinarse, bajo orientación didáctica, en sistemas complejos, con formas de grupo".

¿Son tan tajantes las diferencias, como lo piensa Aebli? Algunas reflexiones acerca de los cuatro puntos mencionados permiten esbozar una respuesta.

El punto a) fue ya analizado en la primera parte ("distanciamiento del maestro").

El punto b) fue también analizado parcialmente allí (sobre los "desplazamientos"). A propósito de él se refiere Aebli a la "estructuración repentina" o al "agrupamiento repentino", a la "producción, siempre rápida y a veces repentina del equilibrio" (Piaget 1947), por medio de la cual caracteriza Piaget la transición de la fase preoperatoria del desarrollo a la operatoria. Conviene distinguir aquí entre i) el logro de una determinada operación, la construcción de su estructura, y u) la transición a la fase del desarrollo, el logro del nivel de desarrollo, al cual pertenece esa operación. En el caso i) la "estructuración repentina" corresponde aproximadamente a la "comprensión iluminada en un momento determinado", de la estructura interna de una operación, que también Aebli reconoce, bajo el nombre de "intuición" (Aebli 1983). En el caso ii) hay que estar de acuerdo con las observaciones de Aebli, de que la construcción de una sola operación no implica necesariamente el logro automático de un nivel estructural. Como se puede deducir de su fórmula analizada en la primera parte, pueden coexistir en determinada fase 
del desarrollo diferentes niveles estructurales. Con esta posición coinciden también los representantes de la psicología evolutiva soviética, quienes sostienen que pueden aparecer formas más primitivas de desarrollo en estadios evolutivos más tardíos ( $\mathrm{y}$, a la inversa, asomarse desde los estadios inferiores alguna estructura correspondiente a los siguientes).

Con respecto a los puntos c) y d) es útil referirnos a las reflexiones de Piaget sobre las relaciones entre lógica y psicología:

- En el modelo piagetiano cada fase del desarrollo mental es formalizada por medio de una estructura (lógica), que no corresponde a la totalidad de las actividades intelectuales y mentales de esa fase, sino sólo a su estado de equilibrio.

- Es necesario pues, al hablar de un estadio evolutivo, distinguir con Piaget entre las formas reales y actuales del pensamiento, y las formas de equilibrio. Las formas actuales del pensamiento tienden, sin embargo, hacia las formas de equilibrio, "aún en el caso de que este equilibrio no se alcance, o se alcance sólo parcialmente":

Es importante en efecto distinguir, a propósito de toda actividad del pensamiento, la cuestión de su estado actual y el problema de las formas de equilibrio hacia las cuales tiende, inclusive cuando este equilibrio no ha sido logrado, o lo ha sido apenas parcialmente (Piaget 1956).

- En otras palabras: en cada nivel coexisten formas de equilibrio con otras más primitivas de pensamiento, que corresponden a situaciones de no-equilibrio. Los psicólogos rusos se refieren a este punto de una manera similar: simultáneamente con el pensamiento conceptual pueden estar presentes en las culturas de los pueblos y en el pensamiento de los individuos tipos de pensamiento pre o seudoconceptual.

- En la fase del pensamiento formal (de la lógica formal) esto significaría que junto a las formas de equilibrio, formalizadas por medio de los grupos y agrupaciones, existen también otras formas más primitivas del pensamiento, que no necesariamente pueden ser formalizadas por medio de tales estructuras de grupo. Con una tal interpretación de la posición de Piaget coincidiría también, en mi opinión, la caracterización que hace Aebli de las operaciones.

- Consecuentemente, también la reversibilidad y la asociatividad, formas más elevadas de la movilidad estructural dentro del pensamiento formal, coexistirían, de acuerdo con esta lectura de Piaget, con otras formas de movilidad.

- El mismo Piaget entiende la reversibilidad y la asociatividad como resultados de procesos prolongados de interiorización y descentración, mediante los cuales la movilidad operatoria se distancia paulatinamente de los puntos de vista aislados y parciales de las actividades perceptivas. A la reversibilidad operatoria y formal preceden otras formas más primitivas de reversibilidad (la reciprocidad, por ejemplo).

Después de proporcionar su propia definición de operación resume Aebli de la siguiente manera en 12 formas... su posición con respecto a la concepción piagetiana:

Puede advertirse que en esta definición no decimos nada acerca de la movilidad y la sistematización de las operaciones. No dudamos que existan operaciones móviles y constitutivas de sistemas, pero opinamos que resulta difícil determinar qué grado de movilidad hay que alcanzar para poder hablar de una operación. Resulta igualmente difícil definir las exigencias que deben satisfacer los sistemas para reconocer como tales a las operaciones cons- 
tituyentes. Piaget, de todos modos, ha planteado tan altas las exigencias que operaciones clásicas, como la sustracción con números naturales, no quedan ya incluidas en su definición de operación, y asimismo la exigencia de reversibilidad resulta difícil de satisfacer en muchas operaciones geométricas. Opinamos, sin embargo, con Piaget, que el hecho de dirigir la atención a la estructura tiene como consecuencia que las operaciones se hacen cada vez más móviles y pueden constituir sistemas de creciente complejidad (Aebli 1988).

La elección del ejemplo de la sustracción no es muy afortunada: esta operación matemática sólo es posible cuando la estructura de los números naturales ha sido construida por medio de la adición. Y precisamente al operacionalizar la adición es posible —apoyándose en la acción correspondiente, pero también en abstracto— volver cada vez al punto de partida. Por eso se contempla en el pensamiento matemático a la sustracción como la adición invertida. $\mathrm{Y}$ eso es precisamente lo que significa la reversibilidad: la posibilidad de regreso al punto de partida (Piaget 1947). Lo cual no significa que la acción correspondiente (como sucede tal vez en las operaciones geométricas o químicas) "de facto" sean reversibles: por esto la operación se halla mediatizada por el proceso de abstracción y el distancia-miento de la percepción.

Por lo que respecta a la pregunta más general de Aebli, qué tan elevado tiene que ser el grado de movilidad, para que se pueda hablar de operaciones genuinas, hay que reconocer que el término operación es algo ambivalente en Piaget. Generalmente emplea el término para referirse a las operaciones formales y caracterizadas por la reversibilidad (inversión) y la asociatividad. Por otro lado le asigna también con frecuencia el significado más amplio de las transformaciones que constituyen las estructuras generales del pensamiento humano. Las primeras operaciones, las genuinas operaciones del pensamiento formal correspondientes al estado de equilibrio de la fase superior del desarrollo, no serían entonces más que un caso particular de las segundas "operaciones", más generales.

Con esta aclaración es explicable el siguiente texto, que muestra cómo el operar, como actividad general del pensamiento, ya se ha desarrollado con anterioridad a la aparición del pensamiento formal.

Se puede ahora afirmar que las primeras operaciones, de las cuales se sirve el niño en su desarrollo y las cuales provienen inmediatamente de las coordinaciones generales de sus acciones sobre los objetos, permiten ser clasificadas en tres grandes categorías, según sea que su reversibilidad se manifieste por medio de la inversión en el tipo de las estructuras algebraicas [...] o por medio de la reciprocidad como en las estructuras de orden [...], o que la articulación no se apoye en semejanzas y desemejanzas, sino que se dé a partir de leyes de vecindad, continuidad y frontera [...] (Piaget 1968a).

Quisiera terminar este aparte diciendo que una segunda lectura del punto crucial "operación", tanto en Piaget como en Aebli, pudiera convencernos de que 1) Aebli no se ha distanciado de la posición piagetiana tanto como lo manifiesta y que 2) Piaget no parece ser tan fixista y substancialista como Aebli quisiera hacerlo aparecer. Movimiento y sistema, "pensamiento estructural y serial", la génesis y la estructura, son las unidades dialécticas que determinan el método de Piaget (Eco). Concentrarse en la una (la estructura) prescindiendo de la otra (la génesis) sólo puede conducir a reducir el pensamiento piagetiano a una falsa caricatura. 


\section{La construcción de los conceptos}

Con los conceptos completa Aebli el proceso estructural de la construcción del pensamiento, iniciado con las acciones y con su forma abstracta, las operaciones. Desde tres diferentes puntos de vista se aproxima a la esencia del "concepto" (Aebli 1983):

1) El contenido: en el concepto se reúnen los "nexos esenciales" de un fenómeno.

2) La instrumentalidad: "Ios conceptos no son sencillamente contenidos de la vida intelectual". "Son los instrumentos que nos permiten ver y comprender el mundo".

3) La funcionalidad: los conceptos son "las unidades, con las cuales pensamos -Se puede por tanto, al pensar, combinar, yuxtaponer o reestructurar conceptos.

Como las acciones y las operaciones, los conceptos son momentos estructurales en la construcción del pensamiento, puntos de cristalización de un proceso. Por esta razón es más importante clarificar su conformación que precisar su definición. Aebli lo intenta usando dos modelos diferentes.

\section{1) El modelo de las características esenciales}

El empleo de este modelo, que no está exento de ciertas dificultades, aparece como un recurso importante en las dos obras de Aebli: con su ayuda introduce el autor la parte psicológica de la formación de conceptos en 12 formas... (Aebli 1983) y a su discusión le dedica una larga sección del segundo tomo de Denken. -(Aebli 1981).

¿En qué consiste el modelo?

Se parte de un conjunto básico cualquiera de objetos, caracterizados por rasgos específicos. Cada rasgo constituye una dimensión (una variable), que puede tener diferentes valores (marcaciones del rasgo). Cada objeto del conjunto básico tiene por lo tanto una marcación específica por cada una de las dimensiones definidas al inicio. El contenido del concepto para un subconjunto definido dentro del conjunto básico consiste en marcaciones determinadas de una parte de las dimensiones originales. A estas dimensiones determinantes se las llama las características esenciales del concepto. La extensión del concepto es el subconjunto de los objetos identificados por medio de dichas marcaciones dimensionales.

Un ejemplo permite aclarar esta concepción: las figuras de un conjunto básico se identifican por medio de cuatro características o dimensiones y sus marcaciones correspondientes:

\begin{tabular}{ll}
\hline Dimensiones & Marcaciones posibles \\
\hline Color: & Rojo, blanco o negro \\
Forma: & Circular, cuadrada o triangular \\
Tamaño: & Grande o pequeño \\
Borde: & Liso o dentado \\
\hline
\end{tabular}


Acto seguido se pueden asignar contenidos conceptuales a diferentes subconjuntos:

Concepto A: "las figuras rojas de forma circular" (extensión), o "el color rojo y la forma circular de las figuras" (contenido). Para este concepto dos características o dimensiones son relevantes o esenciales: el color (rojo) y la forma (circular). Las otras (tamaño, borde) son irrelevantes: figuras grandes o pequeñas, con borde liso o dentado, pertenecen al subconjunto determinado por el concepto.

Concepto B: "las figuras rojas" (extensión) o "el color rojo de las figuras" (contenido). Para este concepto sólo una característica es relevante: el color. El contenido conceptual es por tanto más reducido que en el ejemplo A (menos información), pero la extensión es mayor, puesto que el subconjunto definido en $B$ contiene más elementos que el definido en A: no sólo las figuras rojas de forma circular, sino también las rojas de forma cuadrada o triangular.

Aebli hace uso de este modelo en el ejemplo de los hongos en 12 formas... Se trata de un planteamiento hecho en la forma de solución de problemas, con el cual se pretende formar el concepto "venenosidad-comestibilidad" en los hongos, siguiendo una serie de estrategias:

a) Se definen en primer lugar las características perceptibles más importantes de los hongos, como cinco dimensiones de las cuales cada una puede tener dos valores o marcaciones (características bidimensionales):

- forma general (sombrero o embudo)

- color (claro u oscuro)

- tallo (con o sin verrugas)

- borde (liso o dentado)

- base (con o sin abultamiento)

b) Se clasifican entonces los hongos de acuerdo a las combinaciones posibles de los valores de las dimensiones. En este caso da como resultado $2^{5}=32$ clases de hongos.

c) Se pasa en seguida a la estrategia de planteamiento y comprobación de las hipótesis. Con ello se pretende averiguar qué clases pertenecen al subconjunto "hongos comestibles", y cuáles no (los venenosos): es la extensión del concepto. Y, consecuentemente, qué características constituyen el contenido del concepto. La comprobación de una hipótesis se lleva a cabo degustando en cada caso una partecita de la clase seleccionada de hongos, para saber si es venenoso o comestible.

d) En la práctica no se puede correr el riesgo de someterse a probar todas las 32 clases de hongos. La solución al problema debe ser optimizada en la medida en que con el menor número posible de pruebas se puedan comprobar todas las hipótesis posibles. A fin de eliminar características no esenciales o irrelevantes, se pueden comparar dos pruebas: si las dos son positivas (o negativas) serán por tanto irrelevantes las características cuyas marcaciones cambian. Si ambas degustaciones arrojan resultados diferentes (comestibilidad jvenenosidad) serán entonces irrelevlantes las características cuyas marcaciones no varían. Por medio de tests adicionales se comprueban las dimensiones restantes, eliminando las características no relevantes (eliminación de hipótesis), hasta que sólo queden las relevantes y sus marcaciones (validación de hipótesis). 
El ejemplo citado debe tener como requisito (lo cual no aparece claro en el análisis de Aebli) que las características constituyen de hecho elementos del concepto y que sus valores son multiplicativos. En caso contrario habría que realizar de todos modos las 32 degustaciones para establecer qué clases de hongos son venenosos y cuáles no. Más aún, sería incluso factible suponer que dentro de una misma clase (v.g.: hongos en forma de sombrero, claros, sin abultamiento en la base, con verrugas y borde dentado) se puedan encontrar subconjuntos de hongos venenosos y subconjuntos de comestibles.

En el aparte citado anteriormente de Denken... Aebli resume la historia de la literatura sobre este modelo: parte de Ach, Hull, Wygotski, Hanfmann \& Kasanin, Hovland, hasta la sistematización y construcción de hipótesis en Bruner. En todos los intentos se parte de sistemas cerrados, en los cuales un subconjunto es definido de manera exacta por medio de un número determinado de características con marcaciones específicas. Se trata de investigaciones experimentales en las cuales el sujeto debe desarrollar o seguir estrategias que le permitan descubrir un subconjunto al cual el investigador le ha asignado un concepto.

Aebli concluye que en todos ellos se trata más del "hallazgo" que de la "construcción" de los conceptos. La abstracción que se lleva a cabo no es un proceso constructivo. Las características revelantes están presentes con anterioridad: solamente es preciso aislarlas de las irrelevantes, separar las marcaciones pertinentes de las no pertinentes, rechazar las hipótesis no válidas, a fin de llegar finalmente al concepto deseado.

Los investigadores citados se ocuparon además de conjuntos de objetos abstractos, o sea de una caricatura de la realidad. Etiquetaron los subconjuntos correspondientes a los conceptos con nombres o sílabas sin sentido: "un RAS es, por ejemplo, un cuerpo grande y liviano" (Aebli 1981). Este procedimiento margina de las situaciones vitales y por tanto no representa para Aebli interés alguno para las situaciones escolares. Quizá por eso buscó trasponer el modelo al problema de la venenosidad/comestibilidad de los hongos. Esta transposición no está, sin embargo, exenta de problemas. Mientras que en el ejemplo citado las marcaciones específicas (grande, liviano) de las características revelantes (tamaño, peso) son de hecho componentes del concepto "RAS", no podríamos afirmar lo mismo de las características color y forma en el concepto venenosidad/comestibilidad de los hongos. En este caso las marcaciones de los rasgos representan meramente "indicadores". La venenosidad de los hongos, por ejemplo, puede ser captada o construida como concepto sólo con referencia a la acción correspondiente (real o representada) y a sus relaciones intrínsecas (alguien prueba un hongo, ese alguien se enferma, se da una relación causa-efecto entre ambos sucesos): con ello sólo quiero retomar la teoría constructivista del mismo Aebli.

Hasta aquí se ha trabajado en el modelo con sistemas cerrados en los cuales todas las dimensiones se hallan presentes en todos los objetos, pero solamente la variación de algunas de ellas (las relevantes) determina si un objeto pertenece al subconjunto o no, corresponde al concepto o no. A este sistema contrapone Aebli los sistemas abiertos.

Cita en primer lugar la experiencia sugerida Hull. De entre un grupo de caracteres chinos el sujeto debe encontrar en el experimento, aquellos que tienen algo en común (un rasgo, en la forma de un elemento parcial del carácter) y son identificados con una sílaba. Con frecuencia logra el sujeto el objetivo, aun sin llegar a identificar plenamente la característica. 
En segundo lugar, se refiere a los experimentos de Heidbreder. Los subconjuntos se distinguen entre sí, no gracias a las diferentes marcaciones en las características relevantes, sino por medio de las características mismas. Al sujeto se le pide clasificar las figuras de acuerdo a estas características fundamentales. Por ejemplo, el grupo de las figuras con color, el grupo de las cosas concretas, el grupo de las figuras espaciales, el grupo de los objetos contables, etc.

En 12 formas... Aebli aporta sus propios ejemplos, que deben adecuarse a las situaciones escolares. En todos ellos se trata de identificar los rasgos comunes (una especie de características relevantes cualitativas) a un subconjunto de objetos 0 acontecimientos, para que de esta manera se pueda aproximar el alumno al concepto correspondiente. En una serie de frases debe formar el concepto "sujeto", en una serie de obras maestras el concepto "barroco", en una serie de operaciones matemáticas el concepto "adición", etc.

Aebli encuentra en este tipo de sistemas abiertos todo lo que ha echado de menos en los cerrados. En primer lugar, se trata de situaciones tomadas de la vida real, que se pueden aplicar como ejemplos prácticos en el salón de clase. Se trata, en segundo lugar, de una construcción real de conceptos, en contraposición al hallazgo de conceptos de los sistemas cerrados. En tercer lugar, no se trata ya de eliminación de hipótesis, de aislamiento de características relevantes, sino de la conformación verdadera de las hipótesis, del acercamiento progresivo, gracias a la construcción, a los conceptos correctos. $\mathrm{Y}$, finalmente, no se trata del tipo de abstracción en la cual no se logran conocimientos nuevos, sino de la inducción en el sentido del positivismo de Bacon, único procedimiento capaz de asegurar la construcción verdadera de los conceptos.

A pesar del intento de acercarse a las situaciones vitales por medio de los sistemas abiertos, este modelo sigue siendo estático e incapaz de adaptarse a la psicología constructivista. El concepto, o al menos su contenido, continúa siendo concebido como un conjunto de características. La formación de los conceptos sigue consistiendo en delimitar, por medio de los rasgos comunes, a un subconjunto dentro de un conjunto básico. Los rasgos comunes están presentes desde el principio, independientemente de la actividad humana. La tarea decisiva continúa siendo o bien abstraer del conjunto el subconjunto, o bien definirlo mediante una comparación interminable de objetos. En el fondo continúa vigente la concepción de la realidad como un conjunto enorme de subconjuntos preestablecidos, que pueden ser experimentados solamente a través de sus rasgos comunes. Mucha "contemplación" y poca construcción.

El hallazgo de los conceptos mediante esta clase de abstracción está emparentado con la tradición escolástico-aristotélica, según la cual una definición correcta se logra recurriendo al "genus proximus" y a la "díferentia specifica". Pero Aebli rechaza esa clase de abstracción, puesto que no la considera apta para clarificar el proceso de la formación de los conceptos. Por esta razón tampoco tiene éxito cuando busca trasladarla a una situación de la vida real, como en el caso del problema de los hongos. Pero tampoco la búsqueda de los rasgos comunes en la comparación interminable de objetos, como parece ser el caso de la inducción en los sistemas abiertos, colma las expectativas de la teoría de Aebli. No sin razón había rechazado anteriormente, no sólo la tradición aristotélica, sino también la empírica clásica:

Un sujeto investigado ejecuta esta construcción [de conceptos] por regla general en el estudio intensivo de un caso único y no en la comparación de muchos casos singulares, con objeto de eliminar los rasgos irrevelantes (Aebli 1981). 
Según mi opinión el problema del modelo consiste en concebir al concepto como una colección de rasgos o características, como una contemplación estática de las características esenciales. El mismo Aebli parece llegar implícitamente a esta convicción: después de dedicar largas páginas a la profundización y clarificación de este modelo, deja de tenerlo en cuenta en las consideraciones posteriores sobre la construcción de los conceptos.

\section{2) El modelo de la red}

En 12 formas... se menciona este modelo sólo de una manera superficial, como punto de referencia para la clarificación de la formación de conceptos en las áreas psicológica y didáctica. No obstante, creo que la profundización progresiva en el modelo es la clave para una visión integradora de acciones, operaciones y conceptos, como subprocesos de un proceso único de la construcción del pensamiento. Y este es el tema central de la obra Denken...

De una manera simplificada podrían esquematizarse los pasos siguientes del modelo (Aebli 1981):

a) Una acción simple produce una relación activa entre sus diferentes participantes. En el lenguaje hablado la acción corresponde al verbo, y los participantes son los diferentes casos (siguiendo el modelo de la "gramática de casos" de Fillmore): sujeto, objeto, receptor, instrumento, etc. Consecuentemente puede representarse la acción con un esquema sencillo que indique la relación entre los participantes, con un gráfico, con una proposición centrada en el verbo, del tipo de la lógica preposicional, o con un comando en un programa de computador.

Por ejemplo, la acción simple, "Jorge escribe una carta a María con un lapicero", puede representarse con el esquema:

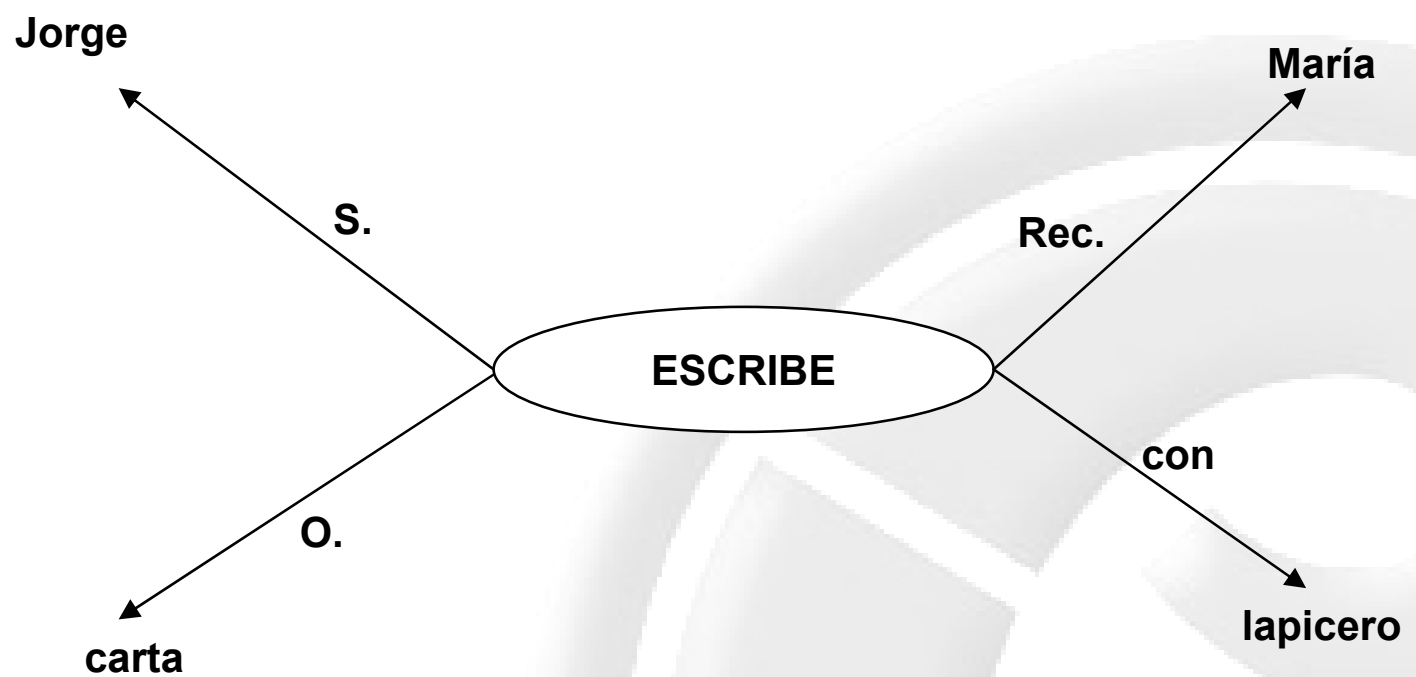

o, más sencillamente, con la proposición:

- ESCRIBE (S: Jorge, O: carta, Rec.: María, con: lapicero).

De esta manera se contemplan los participantes de la acción en relación con, o modificados por la relación activa (representada por el verbo). 
b) Una acción procedimental o de tipo más complejo se compone de una serie de pasos, subacciones o acciones más simples. Cada nueva subacción está ligada con alguna o algunas de las anteriores, en la medida en que se refiere a (tiene como participantes a) participantes modificados en subacciones anteriores, a los resultados o a las relaciones mismas de subacciones anteriores. Este tipo de acción serial o procedimental podrá por tanto ser representada:

- por una cadena de proposiciones, cuyos participantes de la acción (casos) o las relaciones (verbo) pueden ser apelados una y otra vez,

- por una red que enlaza diversos esquemas de acciones simples, cuyas líneas representarían las relaciones creadas en las acciones entre los diferentes participantes, representados por los nudos,

- o más sencillamente, si se complica la red y quiere evitarse la representación abstracta de las proposiciones, por medio de un esquema, o un dibujo, que seleccione las relaciones esenciales, como sucedería en el caso de la explicación del funcionamiento de una máquina complicada.

En los tres tipos de representación se conserva la idea de la red, con sus líneas y sus nudos. Nuevas acciones generan nuevas relaciones entre nudos y otros nuevos (nuevos participantes). De esta manera se almacena el saber actual del hombre: en forma de red. Los objetos, que se encuentran tras los nudos, son captados de manera activa, por medio de las relaciones producidas por las acciones en que toman parte. Son, por decirlo así, conglomerados, entrelazamientos de relaciones.

c) Aebli pasa por alto con frecuencia el papel de las operaciones, cuando se trata de la recapitulación general de su modelo: deduce de manera inmediata la construcción de los conceptos a partir de las acciones, de las relaciones que éstas fomentan. Pero el hecho de conformar esquemas de acción significa que una primera forma de operación ya se halla presente: se desdeña lo "accidental", al concentrarse en las relaciones internas, estructurales, mientras se actúa. De esta manera la red de acciones se convierte progresivamente en una red de operaciones: las relaciones primitivas, algo más superficiales, van siendo integradas en relaciones ulteriores, más internalizadas, más esenciales. Pronto se descubre la posibilidad de alterar o invertir la dirección de estas relaciones dentro de la red (reversibilidad), o de llegar al mismo punto por caminos alternativos (transitividad). Todo ello es posible porque se abstrae (y ello, apoyándose en las acciones correspondientes, reales o representadas) en la red de acciones/relaciones: esto es precisamente lo que Aebli entiende por "operar". La conformación de la red, por lo tanto, solamente es posible en la medida en que se opera. Y operar es una cosa que. se logra paulatinamente (según Piaget, en etapas).

d) La contemplación de cada nudo en la red es la construcción del concepto. Detrás de cada nudo se halla un objeto. De esta manera el concepto señala un objeto detrás de la red. Pero el meollo del concepto no es el objeto, que presta su nombre al concepto, "sino la relación, en medio de la cual aparece el objeto". El concepto tampoco es necesariamente una definición, en sentido de una colección de características o de un "genus proximus" y una "diferentia specifica". En vez de características tenemos acá nudos de relaciones que se construyen de una manera activa.

Resumiendo, los conceptos se construyen de la siguiente manera: en primer lugar, por medio de las acciones se construyen redes de relaciones. En seguida se van 
transformando muchas de estas relaciones en más pocas, más estructurales y más intrínsecas. En tercer lugar se objetiviza un nudo de estas relaciones estructurales por medio de un nombre, un signo. Finalmente, se contempla una red de relaciones próximas desde la perspectiva de ese nudo, objetivizado de la manera descrita; la red se jerarquiza, el nudo se convierte en una cima: ésta es la construcción del concepto.

Gracias al modelo de la red pueden comprenderse fácilmente propiedades adicionales de los conceptos, que aparecen una y otra vez en el trabajo de Aebli:

- Como se dijo arriba, la objetivación del concepto es mediatizada por el nombramiento o señalamiento del objeto que se halla tras el nudo de relaciones. Para ello se desarrolla el proceso de simbolización, en el cual el lenguaje desempeña un papel importante. Este papel del lenguaje, como acompañante de la construcción de los conceptos, había sido subrayado ya por la psicología soviética. Pero nombrar no quiere decir necesariamente definir correctamente el concepto. Un niño (o un adulto) puede haber logrado ya la visión clara del concepto, la concatenación de sus relaciones fundamentales, puede incluso emplear su nombre de manera correcta, aun antes de acertar con una definición adecuada. En la representación pro posicional aludida antes, esto quiere decir que una cadena de proposiciones puede llevar a la construcción correcta de un concepto, sin que necesariamente aparezca al final de ella la proposición predicativa ES/SON, que representa la definición.

- Aunque el concepto significa la paralización momentánea de una red (en el momento de convertirse el nudo en cima), su configuración sin embargo siempre es móvil. En primer lugar, porque por medio de las acciones y de las operaciones se pueden ligar siempre relaciones cada vez más fundamentales y más nuevas a un nudo. $Y$, en segundo lugar, porque al interior de una red se puede cambiar de cima y reformular nuevos conceptos, correspondientes a otros nudos.

- Lo cual significa que la jerarquización de la red, que tiene lugar en el proceso de construcción de conceptos alrededor de un nudo de relaciones, no tiene nada que ver con la jerarquía estática de un nudo ordenado y definido por medio de los rasgos esenciales fijos del ser, en la tradición escolástica (como en el árbol de Porfirio).

- En la representación proposicional la objetivación del concepto corresponde al predicado o argumentación de una proposición. La proposición, el concepto, va más allá de la simple señalización del producto, ya que es el resultado de un proceso de construcción. Aebli afirma siguiendo a Marx: "afirmamos que en el producto yace verdaderamente el trabajo de su elaboración, que el trabajo está en él de una forma objetivizada, 'materializada' ".

Los conceptos son para Aebli no solamente el resultado del proceso del pensamiento, sino también los instrumentos de la vida mental, los medios con los cuales la persona puede contemplar el mundo. Y la construcción de estos instrumentos está condicionada, de un lado, por el estado de su evolución mental (su capacidad para actuar y, actuando, para operar) y, del otro, por la situación cultural, el medio ambiente, en el cual actúa. A manera de ejemplo, sigamos la construcción del concepto "madre" en contextos hipotéticos culturales y personales diversos, esto es, en redes de relaciones distintas, de acuerdo al modelo de Aebli: 
a) Para un niño pequeño madre es la persona que "está a mi lado, que se preocupa por mí". Tal tipo de seudo o preconcepto se construye en una red de relaciones centradas en el actuante. La representación proposicional de la red sería:

1. ESTA $_{\text {cerca }}$ (mujer, yo)

2. SE-PREOCUPA (mujer1, yo)

3. ES (madre, mujer2)

b) Desde la perspectiva cultural de la familia tradicional, ser-madre tiene un significado de relación biológica, que generalmente se halla mediatizado por las relaciones vivirjuntos y estar-casados. En este caso el concepto madre se construye paralelamente al concepto padre, en la red siguiente:

1. ESTAN-CASADOS (hombre, mujer)

2. VIVEN-JUNTOS (hombre1, mujer 1)

3. TIENEN- RELACIONES-SEXUALES (hombre2, mujer2)

4. DA-A-LUZ (mujer3, niño, de: hombre3)

5. CAUSA-EFECTO (prop. 3, prop. 4)

6. ES (madre, mujer4, de: niño4)

c) Una perspectiva puramente biológica acentuará aquellas relaciones, que en la red anterior se hallaban un poco mediatizadas por los condicionamientos culturales:

1. TIENEN- RELACIONES-SEXUALES (hombre, mujer)

2. DA-A-LUZ (mujer1, niño, de: hombre1)

3. CAUSA-EFECTO (prop.1, prop.2)

4. ES (madre, mujer2, de: niño2)

d) Finalmente, las relaciones pueden también centrarse en el punto de vista de las acciones de la madre soltera. Aquí pueden acentuarse también otras acciones biológicas, y no necesariamente se construye paralelamente el concepto de padre:

1. TIENE-RELACIONES-SEXUALES (mujer, con: $x$ )

2. QUEDA-EMBARAZADA (mujer1)

3. CAUSA-EFECTO (prop.1, prop.2)

4. DA-A-LUZ (mujer2, niño)

5. CAUSA-EFECTO (pro.2. prop.4)

6. ES (madre, mujer4, de: niño4)

\section{TERCERA PARTE: ALGUNAS OBSERVACIONES CRITICAS}

\section{A. Teoría y Praxis}

Según Aebli, existen dos métodos posibles para la formación de maestros (Aebli/Steiner). El primero consiste en tomar como punto de partida los problemas claves que se presentan hoy con más frecuencia en la práctica escolar, sintetizarlos y analizarlos posteriormente a la luz de los principios pedagógicos; la didáctica se construye en este caso a partir de la práctica de los procesos educativos, y se sistematiza después teóricamente. El segundo método parte de un análisis sistemático de las áreas principales de las ciencias de la educación (pedagogía, sociología, pedagógica, filosofía) y busca su aplicación posterior en la práctica escolar; la didáctica se construye en este caso como una disciplina teórica primero, y luego como una disciplina aplicable. 
Para Aebli el mejor método es una complementación de los dos caminos: "de esta manera vemos la formación de maestros ubicada en un campo intensivo de tensión entre teoría y práctica, entre sistematización y aplicación" (Aebli/Steiner).

De una manera similar analiza Schroeter más tarde el origen de la enseñanza didáctica de Aebli. Para aquél la psicología no puede ser un punto de partida autónomo para el desarrollo de una didáctica. La psicología sirve más bien de marco teórico, a partir del cual pueden deducirse conclusiones generales de tipo didáctico en la praxis pedagógica concreta. El mismo Aebli parece creer que muchas de sus disquisiciones didácticas son atribuibles a su confrontación teórica con la escuela piagetiana, cuando éstas en realidad sólo fueron posibles a partir de su experiencia con la actividad docente:

[...] puesto que - como se dijo antes- Aebli cree (posiblemente a consecuencia de sus esquemas personales de asimilación) que las conclusiones didácticas mencionadas por él surgen exclusivamente de la teoría de Piaget, cuando éstas en verdad debieron haber surgido en parte como productos de la interacción entre sus propios esquemas y la teoría de Piaget (Schroeter).

Podríamos por tanto caracterizar el desarrollo de la teoría didáctica de Aebli como una interacción dialéctica entre la escuela de Ginebra (teoría) y su trabajo concreto en la formación de maestros (praxis), y resumirla en los siguientes pasos:

- Aebli es un maestro (como tal es reconocido por Piaget) que viene a formar-se en la escuela de Ginebra: la praxis busca claridad en la teoría.

- Aebli se apropia de la teoría piagetiana. Como consecuencia busca un camino para aplicar la teoría de la sicología genética en una didáctica (Aebli 1951): aplicación de la teoría en la praxis.

- Esta aplicación práctica es pronto rechazada como insuficiente. Aebli se distancia de la escuela ginebrina y busca confrontar la teoría genética, por un lado, con otras teorías (especialmente con la sicología cognitiva norteamericana) y, por otro, con las preocupaciones didácticas concretas surgidas en la formación de maestros.

- El resultado es un desarrollo cualitativo ulterior de la teoría inicial (Denken: das Ordnen des Tuns), que está en posibilidad de proporcionar elementos fundamentales para sistematizar una didáctica general y aplicable (12 formas básicas de enseñar).

Por consiguiente se puede decir que la didáctica de Aebli sólo es posible, en la medida en que se deriva de la teoría de Piaget; pero, igualmente, no hubiera podido desarrollarse, si no se da la confrontación con la misma a partir de la praxis.

\section{B. Dos modelos complementarios}

Steiner menciona al "ejercicio operatorio" como la clave para la aplicación práctica de la didáctica de Aebli. En él busca el maestro trabajar móvilmente y a fondo operaciones con los alumnos, para que éstos puedan aprender y manejar las estructuras correspondientes:

- "móvilmente" quiere decir, teniendo en cuenta la posibilidad de reversión de la operación (reversibilidad) 
- y quiere decir también, que se pueden encontrar distintos caminos para llegar a la misma meta (asociatividad);

- "trabajar a fondo" no significa que una operación ha de ser aplicada en una serie interminable de casos, sino más bien que "las operaciones se trabajan a fondo en un ejemplo, es decir, se realizan en diversas interconexiones y generando relaciones de maneras diversas, y se integran con ello en una estructura cognitiva" (Steiner).

Este ejercicio operatorio se refiere, según Steiner, a la transición del pensamiento preoperatorio al operatorio, en particular al aprendizaje de las estructuras operatorias en la edad escolar. Si ello es así, entonces habría que añadir que la teoría didáctica de Aebli, que tiene su núcleo teórico en la tríada constructivista acciones-operaciones-conceptos, y el práctico en el ejercicio operatorio, tendría validez únicamente para la fase de maduración, cuando se logra el pensamiento formal, y no para el conjunto del proceso de aprendizaje, en todas las fases. Si bien esta fase de transición es la más importante, didácticamente hablando, puesto que a ella corresponde la mayor parte de la edad escolar, no obstante

a) no se encuentra indicio alguno en la obra de Aebli de que la didáctica sugerida por él no pueda aplicarse a otra edad más temprana, y

b) también parece reconocer que formas más primitivas de pensamiento aparecen todavía en edades posteriores (los psicólogos rusos habían llamado la atención también a este fenómeno, y señalado, entre otros ejemplos, el evolucionar diario del lenguaje).

¿Cómo podrían manejarse entonces didácticamente estas formas de pensamiento más primitivas, si el ejercicio operatorio sólo puede realizarse en las últimas fases del desarrollo del pensamiento?

Propongo una visión alternativa. Creo que, efectivamente, el proceso actuar/ operar/formar-conceptos representa la construcción general del pensamiento, y es reconocible en cualquier estadio de la inteligencia humana, bajo formas diferentes. Para aclararlo quisiera a continuación reestructurar en alguna manera el modelo de Aebli, teniendo en cuenta los hallazgos de Piaget.

Partamos de que el proceso de Aebli,

\begin{tabular}{|ccc|}
\hline 1 & 2 & 3 \\
actuar $\ldots \ldots$ & operar $\ldots \ldots$ & formas conceptos \\
\hline
\end{tabular}

representa tres momentos encadenados uno a otro de cualquier actividad humana inteligente. Se trata en el caso de Aebli, por tanto, de una representación de tipo más bien sincrónico.

En este sentido el pensamiento se realiza en cada estadio gracias a que el actuante se distancia de cierta manera de su acción (abstracción), establece determinadas relaciones (operación) que le permiten manejar cognitivamente su medio (conceptos).

¿En qué se diferencia entonces cada estadio del desarrollo? Según Piaget, en la estructura correspondiente: éstas pueden tomar la forma de ritmos, regulaciones 
(estructuras topológicas y de orden), grupos y agrupaciones. A su vez, cada estructura se caracteriza por un tipo específico de "transformación": repeticiones rítmicas, transformaciones en el tiempo, series, clasificaciones, movimientos espaciales, operaciones matemáticas y lógicas. En consecuencia, podríamos decir que el nivel de desarrollo se caracteriza también por el tipo de transformaciones que ocurren en el.

A estas transformaciones las llamamos provisoriamente el "área operatoria", que corresponde al significado amplio atribuido por Piaget al término "operación"4. El desglose de esta área operatoria en los diversos tipos de transformaciones u operaciones, de los cuales el último correspondería a las operaciones genuinas, "lógicas" (en el sentido estrecho del concepto), permite representar consecuentemente el modelo de Piaget como uno de tipo más bien diacrónico,

\section{Área operatoria}

\begin{tabular}{l|l|} 
Estadio 1 & Tipo 1 de operaciones \\
\hline Estadio 2 & Tipo 2 de operaciones \\
\hline Estadio 3 & Tipo 3 de operaciones \\
\hline Estadio 4 & Tipo 4 de operaciones
\end{tabular}

\section{tiempo}

Esta área operacional estructurada en estadios (la representación diacrónica de Piaget) corresponde al segundo momento de la representación sincrónica de Aebli. Podemos pues entrelazar los dos modelos en el siguiente esquema:

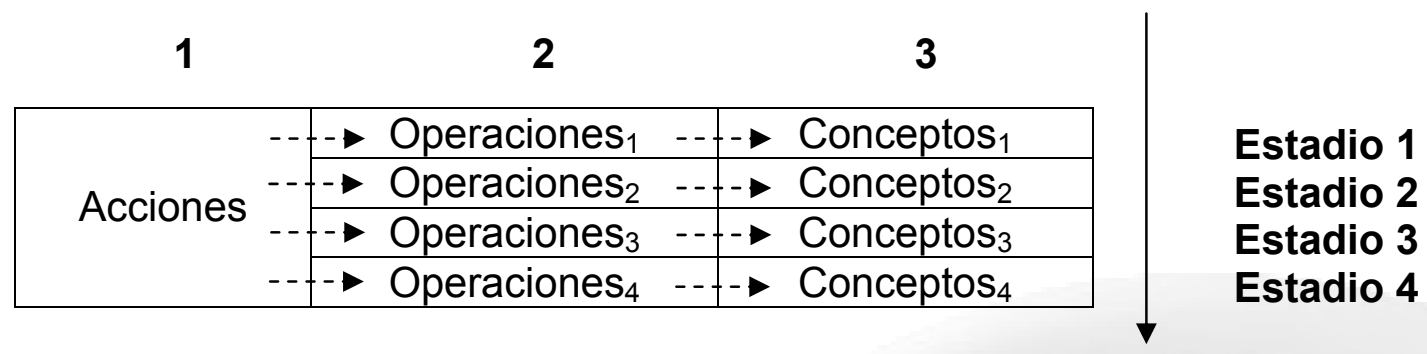

con las siguientes anotaciones:

- Las acciones fueron representadas por medio de un continuo, porque se trata de la interacción más externa e inmediata con el medio, y por lo tanto tienen validez como punto de partida para la vida mental en todos los estadios. Lo que no significa que el actuar no evolucione cualitativamente, por ejemplo en la dirección de acciones cada vez más interiorizadas, más representadas, o más complejas.

- Anteriormente vimos cómo los conceptos son construidos a partir del operar. La variación en la manera de operar determinará igualmente una variación correspondiente a la captación del mundo, en los conceptos. Por consiguiente, se podrían caracterizar los diferentes niveles no solamente por la clase de operaciones y estructuras operacionales, sino también por la clase de conceptos que se forman en estas estructuras cognitivas: el último estadio corresponde al pensamiento formal, con las operaciones formales (reversibles) y los conceptos "genuinos" correspondientes. Los estadios anteriores son por tanto (siguiendo a Piaget) fases del pensamiento preoperatorio, caracterizados por sistemas más elementales de operaciones (regulaciones, operaciones prelógicas) y por los seudoconceptos y preconceptos correspondientes.

\footnotetext{
${ }^{4}$ Véase la segunda parte, B. 3, "las operaciones de Aebli frente a las operaciones de Piaget". 
Piaget caracterizó los estadios del desarrollo por medio de las operaciones y de sus estructuras, Wygotski por medio de los conceptos. Ambos puntos de vista son por tanto complementarios. Según mi opinión, la caracterización de Piaget es más dinámica y más cercana al constructivismo, puesto que las operaciones son procesos activos, mientras que los conceptos solamente son sus resultados provisionales (con ello coincide también la crítica que hace Galperin a Wygotski).

- Los estadios no son simplemente casillas aisladas, yuxtapuestas. Con ello se aclara por qué, según opinión de Aebli, diversos factores pueden hacer que estructuras operatorias más primitivas aparezcan en fases posteriores, y por qué, según opinión de Wygotski, pueden aparecer seudoconceptos en el pensamiento conceptual de los adultos.

- La construcción del pensamiento está representada mediante el progreso en las dos direcciones del gráfico: horizontal (de izquierda a derecha) y vertical (de arriba a abajo). La actividad didáctica (el ejercicio operatorio) debería entonces proporcionar al alumno la oportunidad de desarrollarse en ambos sentidos:

a) Horizontalmente, para que actúe mejor, para que a partir de la acción pueda construir las operaciones correspondientes a su nivel de desarrollo y los conceptos con los cuales maneje su medio. Se trata de los procesos de la abstracción (transición de la acción a la operación) y de la jerarquización (transición de la operación al concepto), que le permiten la construcción de estructuras de totalidad en un estadio dado del desarrollo.

b) Verticalmente, para que esté en capacidad de integrar las estructuras ya alcanzadas en un nivel superior. Se trata del proceso de la maduración, con el cual se acerca al pensamiento lógico.

Este movimiento vertical de "maduración" se da no solamente en el tránsito de un estadio a otro, sino también dentro del mismo estadio, ya que en él pueden coexistir niveles estructurales de complejidad y poder integrador diversos. Con ello pueden rechazarse ciertas interpretaciones corrientes del pensamiento piagetiano, según las cuales el desarrollo mental terminaría con el acceso a las operaciones formales (11/12 14 años de edad): por un lado, como hemos visto, la aparición de dichas estructuras no reviste necesariamente un carácter definitivo; por otro lado, el mismo Piaget reconoce el carácter más asindético que permanente de los niveles de equilibrio; y, en tercer lugar, la movilidad dentro del pensamiento lógico implica que puedan aparecer siempre nuevos y más elevados niveles de formalización, nuevas y más comprensivas metaoperaciones (como ocurre, por ejemplo, en el pensamiento científico).

Para precisar con más exactitud estas dos direcciones del trabajo didáctico habría que llenar de contenido el esquema aportado. El siguiente gráfico no es más que un intento de insinuar sus rasgos más generales: 
estadio

\begin{tabular}{|lll|}
\hline $\begin{array}{l}\text { Pensamiento } \\
\text { Sensomotor }\end{array}$ & $\begin{array}{l}\text { Ritmos: } \\
\text { Movimientos } \\
\text { Repeticiones }\end{array}$ & $\begin{array}{l}\text { Objetos sentidos, } \\
\text { Percibidos }\end{array}$ \\
\hline $\begin{array}{l}\text { Pensamiento } \\
\text { Preoperatorio }\end{array}$ & $\begin{array}{l}\text { Regulaciones: } \\
\text { Estructuras } \\
\text { Topológicas, } \\
\text { De orden }\end{array}$ & $\begin{array}{l}\text { Objetos nombrados } \\
\text { Seudocenceptos }\end{array}$ \\
\hline $\begin{array}{l}\text { Operaciones } \\
\text { Concretas }\end{array}$ & $\begin{array}{l}\text { Op. Concretas: } \\
\text { Agrupaciones } \\
\text { grupos }\end{array}$ & (pre) conceptos \\
\hline $\begin{array}{l}\text { Pensamiento } \\
\text { Formal }\end{array}$ & $\begin{array}{l}\text { Sistema operatorio } \\
\text { Lógico }\end{array}$ & conceptos \\
\hline
\end{tabular}

De acuerdo con este esquema el "ejercicio operatorio" consistiría en ejercitar, según el estadio, los ritmos, las regulaciones, las operaciones concretas o formales, para que el niño a) desarrolle los instrumentos correspondientes (acciones, operaciones, conceptos) para el ordenamiento activo y la comprensión del mundo (movimiento horizontal), y b) pueda acceder posteriormente al estadio siguiente (movimiento vertical). Aunque, hay que repetirlo de nuevo, el "ejercicio operatorio" permanece primordialmente en el campo de los sistemas de operaciones y de la formulación de conceptos genuinos, o en la transición al pensamiento formal, puesto que la mayor parte de los alumnos se encuentran en las dos últimas etapas del desarrollo mental.

\section{La fundamentación filosófica}

Al final del segundo tomo de Denken: das Ordnen des Tuns Aebli presenta la relación que existe entre el pensamiento (tema central del libro) y el aprendizaje, el desarrollo y la educación (Aebli 1981):

a) Pensamiento implica para Aebli la construcción de nuevas estructuras comportamentales. Significa por tanto modificación del comportamiento, y esto es aprendizaje.

b) Tales estructuras del pensamiento son cada vez más complejas. El pensamiento estructural logra por tanto niveles cada vez más nuevos y elevados. Se trata del desarrollo.

c) Este proceso mental tiene lugar no sólo de manera espontánea, sino también dirigida. Las estructuras del pensamiento se logran por lo tanto por medio de la educación.

La clave de esta relación triple es la concepción estructural del pensamiento, a) como construcción, es decir como logro de la actividad del sujeto, y b) al mismo tiempo, y a diferencia de Piaget, de manera dirigida. Educación es por tanto la construcción dirigida, orientada, de las estructuras mentales (del pensamiento), por tanto también del aprendizaje y del desarrollo. Todo ser humano construye sus propias estructuras mentales y lo hace mediante la interacción social. 
El pedagogo puede entonces preguntarse por la consistencia de estas estructuras: ¿son diferentes para cada hombre, para cada sociedad? ¿O se trata siempre de las mismas estructuras, en cuyo caso construir no significa otra cosa que construir imitando? En otras palabras: ¿se repiten siempre las mismas estructuras en la construcción del pensamiento, en la evolución de la vida mental? ¿Cuál es el modelo? ¿Cómo se produce entonces la evolución del pensamiento social? ¿Existe de antemano un orden preestablecido hacia cuyo reencuentro tiende siempre el ser humano? ¿O significa cada fase evolutiva algo radicalmente nuevo, no solamente en la vida mental del hombre individual, sino también en la historia de la sociedad?

La respuesta a estos interrogantes puede surgir únicamente dentro del contexto de una toma de posición filosófica frente al ser del hombre y de la sociedad. Pero una posición filosófica es ya una visión del mundo y sólo puede lograrse, según la teoría de Aebli, a partir de las acciones, de la interacción con el medio concreto, de las actividades prácticas del que filosofa.

Aebli cree ver el origen de su constructivismo en su procedencia como persona de un ambiente suizo, calvinista y liberal (Aebli 1981). Dentro de este contexto político, cultural, económico y social, considera al pragmatismo como la característica más esencial de su pensamiento. Un pragmatismo que deriva el pensamiento de la actividad, lo mental de lo material. Pero, hay que añadir, pragmatismo que se mueve así mismo dentro de las reglas de juego de una cultura occidental, industrializada, cristiana. Pragmatismo, por tanto, que implica continuidad de las reglas de juego imperantes, más que cambios estructurales de las mismas.

Dentro de esta continuidad liga su credo filosófico con la filosofía de Kant. La asimilación según Piaget, y también la del mismo Aebli, estarían emparentadas con el "apriorismo" kantiano, pero concebido de una manera más dinámica y genética (Aebli 1981). Ello aclara el surgimiento de las estructuras mentales que construye el hombre en su encuentro con el medio: dichas estructuras provienen de esquemas de asimilación; sus elementos están disponibles con anterioridad en el repertorio de la vida mental. Las estructuras ya están presentes, en germen; sólo es necesario desdoblarlas de adentro hacia afuera.

Con su kantismo se distancia Aebli en sus presupuestos filosóficos de los de Piaget (Piaget 1947). Para éste la adaptación de la inteligencia es una clase de interacción dialéctica entre los factores internos y los externos, por tanto una direccionalidad doble, del sujeto hacia el medio y del medio hacia el sujeto. La adaptación no consiste sólo de asimilación: ésta se complementa con el proceso de acomodación.

Con esta contemplación unilateral del kantismo parece caer Aebli finalmente en una metafísica. Si todas las estructuras existen ya en el interior, entonces la búsqueda del orden, es decir el proceso, se convierte en un orden, es decir en un orden preestablecido al proceso. Con tales pensamientos termina la obra Denken: das Ordnen des Tuns: el orden aparece como un orden cuasi-divino de la cultura occidental, algo que está antes de nosotros y hacia el cual tendemos de nuevo. Este orden ya no lo construye uno: "se construye en nosotros....". Uno actúa, pero actúa en un mundo determinado, según las reglas de juego de una sociedad que se mira como el centro del mundo. Desaparece la posibilidad de alterar esas reglas que regulan su orden. Permanece sólo la posibilidad de construir siempre de nuevo el mismo tipo de orden, en el cual cada persona debe contentarse con aceptarlo o, a lo sumo, sólo con mejorarlo en una dirección específica. 
Pragmatismo, kantismo y una especie de metafísica son entonces las tres posiciones teóricas fundamentales que dan piso filosófico al constructivismo de Aebli. Ello es así porque el teórico es también un hombre actuante, que construye su concepción del mundo (es decir su teoría) a partir de su interacción con él, con su propia cultura y su propia situación.

En contextos diferentes, como el nuestro, creo que se puede elaborar también un pensamiento didáctico dentro de los marcos básicos del constructivismo formulado por Aebli. Pero habría que hacer con la teoría de Aebli lo que éste, a su vez, hizo con la teoría piagetiana: distanciarse de ella para, de una manera constructiva, reelaborarla a partir de su confrontación con la práctica pedagógica concreta.

\section{REFERENCIAS BIBLIOGRAFICAS}

\section{a) Obras de Hans Aebli citadas en este trabajo ${ }^{5}$}

(1951) Application ti la didac tique de la psychologie de Jean Piaget, Neuchatel, Delachaux \& Nestlé. Versión española: Una didáctica fundada en la psicología de Jean Piaget, Buenos Aires, Kapeluz, 1973.

(1961) Grundformen del Lehrens [formas básicas de enseñar], Stuttgart, Klett.

(1963), Uber die geistige Entwicklung des Kindes [desarrollo mental del niño], Stuttgart, Klett.

(1969), "Zür Einfúhrung" [introducción], en Ergebnisse der sowjetischen Psychologie, Stuttgart, Klett.

(1980) Denken: das Ordnen des Tuns [el pensamiento como el ordenador del hacer]. Tomo I: Kognitive Aspekte der Hand-lungstheorie [aspectos cognitivos de la teoría de la acción], Stuttgart, Klett-Cotta.

(1981) Denken, das Ordnen des Tuns. Tomo II: Denkprozesse [procesos mentales], Stuttgart, Klett-Cotta.

(1983) Zwölf Grundformen des Lehrens, Stuttgart, Klett-Cotta. Versión española: 12 formas básicas de enseña); Madrid, Narcea, 1988.

(1987) Grundlagen des Lehrens, Stuttgart, Klett-Cotta. Versión española: Factores de la enseñanza que favorecen el aprendizaje autónomo, Madrid, Narcea, 1991.

Aebli, Hans/Steiner, Gerhard (1975) Probleme der Schuylpraxis und der Erziehungswissenschaften [problemas de la práctica escolar y de las ciencias de la educación], Stuttgart. Klett.

\section{b) Obras consultadas de Jean Piaget}

(1947) La Psychologie de l'intelligence, París, Alcan.

\footnotetext{
${ }^{5}$ Las citas del texto (autor y fecha) se refieren a la primera edición, en alemán, de la obra, con el fin de situarlas cronológicamente; aunque, en el caso de las obras traducidas al español, la cita se haya tomado de la traducción.
} 
(1956) "Les Activités Mentales en Rapport avec les Expressions Symboliques Logiques et Mathèmatiques", en Synthese 10, 127-145.

(1962) "Zum Geleit" [prefacio de Aebli 1951, que aparece por primera vez en la primera edición alemana de 1962].

(1968a) Le structuralisme, París, Presses Universitaires de France.

(1968b) "A Theory of Development" en International Encyclopedía of the Social Sciences, vol. 4, New York, Macmillan, 140-147.

(1970) "Piaget's Theory", en Carmichael's Manual of Child Psychology, vol. 1, New York, John Wiley \& Sons.

c) Obras de la psicología soviética consultadas

Galperin, P.J. (1969). "Die Entwicklung der Untersuchungen über die Bildung geistiger Operationen" [desarrollo de las investigaciones sobre la formación de las operaciones mentales], en Eergebnisse der sowjetischen Psychologie, Stuttgart, Klett, 367-405.

Kostkjuk, G.S. (1969) "Fragen der Denkpsychologie" [interrogantes de la psicología del pensamiento ], en Ergebnisse der sowjetischen Psychologie, Stuttgart, Klett, 241-366.

Tjoplenkaja, Ch. N. (1973) "Zum Problem der Begriffsbildung bei Kindern" [el problema de la formación de conceptos en los niños], en Sowjetische Beiträge zur Lerntheorie, Colonia, Pahl-Rugenstein, 41-70.

Wygotski, Lew S. (1974) Denken und Sprechen [pensamiento y lenguaje], Frankfurt, S. Fischer.

\section{d) Otros}

Eco, Humberto (1972) Einführung in die Semiotik [introducción a la semiótica], München, Wilhelm Fink.

Montada, Leo (1970) Die Lernpsychologie Jean Piagets [la psicología del aprendizaje de Jean Piaget], Stuttgart, Klett.

Schhroeter, Gerhard (1981) Psychologische Grundlagen der Didaktik [fundamentos psicológicos de la didáctica], Stuttgart, Klett.

Steiner, Gerhard (1973) Mathematik als Denkerziehung [las matemáticas como educación del pensamiento], Stuttgart, Klett. 